INPLASY

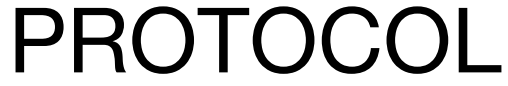

To cite: Muniandy et al. A Meta-Synthesis on Clients' Experience with Tele-

Rehabilitation. Inplasy protocol 202180047. doi:

10.37766/inplasy2021.8.0047

Received: 12 August 2021

Published: 12 August 2021

Corresponding author: Yughdtheswari Muniandy

eshwari_physiorehab@yahoo.com

Author Affiliation:

University Putra Malaysia.

Support: No.

Review Stage at time of this submission: Data analysis.

Conflicts of interest:

None declared.

\section{A Meta-Synthesis on Clients' Experience with Tele-Rehabilitation}

Muniandy, $\mathrm{Y}^{1}$; Romli, $\mathrm{MH}^{2}$; Karuppiah, $\mathrm{K}^{3}$; Manaf, $\mathrm{H}^{4}$.

Review question / Objective: Tele rehabilitation is a subject that contributes to a qualitative approach. The usage of telerehabilitation has a remarkable increase which gives rise to explore the perception of clients using telerehabilitation. For establishing key elements of the review question, we use a PICO (Population, Intervention, Comparison, and outcome) framework (Cooke et al., 2012). The population in the review comprises clients including patients and caregivers who undergo rehabilitation using telerehabilitation services, the intervention involves physiotherapy, occupational therapy, speech or medical rehabilitation, comparison with conventional rehabilitation (face to face) and outcome includes perception and experience of clients. The complete review question was "What is the experience and perception of the client using telerehabilitation?

INPLASY registration number: This protocol was registered with the International Platform of Registered Systematic Review and Meta-Analysis Protocols (INPLASY) on 12 August 2021 and was last updated on 12 August 2021 (registration number INPLASY202180047).

\section{INTRODUCTION}

Review question / Objective: Tele rehabilitation is a subject that contributes to a qualitative approach. The usage of telerehabilitation has a remarkable increase which gives rise to explore the perception of clients using telerehabilitation. For establishing key elements of the review question, we use a PICO (Population, Intervention, Comparison, and outcome) framework (Cooke et al., 2012). The population in the review comprises clients including patients and caregivers who undergo rehabilitation using telerehabilitation services, the intervention involves physiotherapy, occupational therapy, speech or medical 
rehabilitation, comparison with conventional rehabilitation (face to face) and outcome includes perception and experience of clients. The complete review question was "What is the experience and perception of the client using telerehabilitation?

Condition being studied: The use of telerehabilitation as a part of rehabilitation has been popular, empowering health care providers to provide distance support, evaluation and intervention for individuals with disabilities. Areas where rehabilitation providers are in great demand or where specialists are unavailable are driving the need for tele-rehabilitation growth. Several recent publications have addressed the patients' feedback in improvement efforts. These studies have also addressed the barrier and facilitators to the use of digital technologies. Understanding the possibilities of improvement in telerehabilitation services, will overcome the challenges and recognizing the possibility of change of tele-rehabilitation services.

\section{METHODS}

Search strategy: Two reviewers independently screened all publications, including titles, abstracts, and full-text papers. The search was conducted till 27th August 2020. The followings are the search strategy used: Primary search; \#1 "telerehabilitation OR "tele-rehabilitation" OR telehealth OR "tele-health" OR telemedicine OR "tele-medicine" OR telepractice OR "tele-practice" OR telecare OR "tele-care" OR "tele-monitoring" OR telemonitoring; \#2 "qualitative OR "focus group discussion" OR "grounded theory" OR ethnography OR phenomenology OR triagulation OR interview*; \# 3 "rehabilitation OR "occupational therap*" OR "physical therap*" OR "physiotherap*" OR "speech therap*" OR "speech language patholog*"; \#1 and \#2 and \#3 Secondary Search: Manual searching by reviewing the reference list of included studies.

Participant or population: We will only include studies which involves clients including patients and caregivers who undergo rehabilitation using tele-rehab service.

Intervention: Rehabilitation involves speech rehabilitation, occupational therapy, physiotherapy or medical rehabilitation.

Comparator: Comparison with conventional rehabilitation (face to face).

Study designs to be included: Qualitative studies.

Eligibility criteria: To be considered in this review, each study has to meet the following inclusion criteria (1) qualitative study; (2) focus on the experience of clients in using teleconference application for rehabilitation; (3) the study is exploring rehabilitation-related topic (i.e. rehab medicine, occupational therapist, physiotherapist \& speech pathologist); (4) clients including patients and caregivers. Meanwhile, the exclusion criteria are (1) studies investigate on mobile apps; (2) nonEnglish articles; (3) studies in the grey literature (thesis, book, and conference); (4) studies involve monitoring and assessment (as our focus is to understand the merit and demerits using rehabilitation services).

Information sources: An extensive systematic literature search was conducted on 27th August 2020 using eight electronic bibliographic databases; Academic Search Complete, CINAHL, Health Business Elite, MEDLINE, Psychology, and Behavioral Sciences Collection, SPORTDiscus, Scopus, ASEAN Citation Index (ACI) with the full-text original article.

Main outcome(s): Experience and perception of the client towards telerehabilitation.

Data management: Comprehensive data extraction will be performed. A data extraction form will be used for documenting the extracted information. The extracted information includes information on author, year, the objective of the study, characteristics of participants (patients/caregivers), type of qualitative 
framework, type of tele-rehab and protocol, country, and summary findings of the study.

Quality assessment / Risk of bias analysis: The methodological quality of the included studies will be assessed using Crowe Critical Appraisal Tool (CCAT). Two authors have independently assessed the methodological quality of the studies, and disagreements between the review authors will be resolved by discussion, with the involvement of a third review author when necessary.

Strategy of data synthesis: We will provide a narrative synthesis of the findings from the included studies. Once data are obtained, a sheet will be made to tabulate data according to the population of the study, design of the study, and usage of tele-rehabilitation.

Subgroup analysis: A subgroup analysis will be done according to the type of included studies and available data.

Sensitivity analysis: Not conducted.

Language: Limited to English.

Country(ies) involved: Malaysia.

Keywords: Tele-rehabilitation; metasynthesis; perception; protocol.

Dissemination plans: The results of this meta-synthesis will be disseminated by publishing the manuscript in a peerreviewed journal or presenting the findings at a relevant conference.

Contributions of each author:

Author 1 - Yughdtheswari Muniandy.

Email: eshwari_physiorehab@yahoo.com

Author 2 - Muhammad Hibatullah Romli Involved in Formal screening of search results, data extraction, quality assessment, data analysis, data synthesis, and manuscript writing.

Email: mhibatullah@upm.edu.my

Author 3 - Karmegam Karuppiah - Critical analyzing, verifying the results, and writing the manuscript.

Email: megam@upm.edu.my
Author 4 - Haidzir Manaf - Critical analyzing, verifying the results, and writing the manuscript.

Email: haidzir5894@uitm.edu.my 